\title{
Effect of Hypertonic Saline on Survival in an Experimental Embolic Model of Massive Cerebral Infarction with Severe Brain Swelling
}

\author{
Yi Chia Wang, M.D., Thomas JK Toung, M.D., Chi-Hsaing Huang, M.D. \\ Department of Anesthesiology, National Taiwan University Hospital \\ Department of Anesthesiology and Critical Care Medicine, Johns Hopkins University
}

\section{Survival rate in embolic stroke rats model}

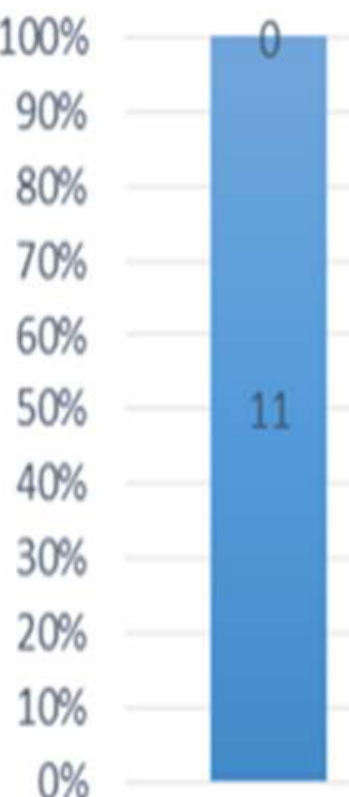

Naïve

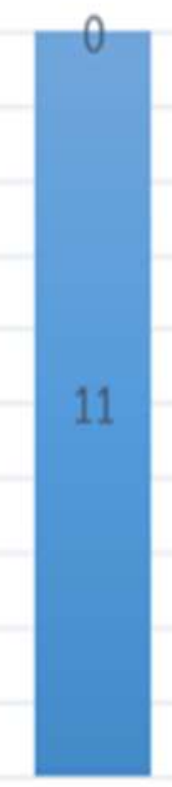

Surgical Sham

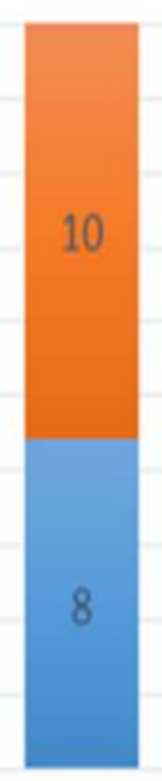

NS control

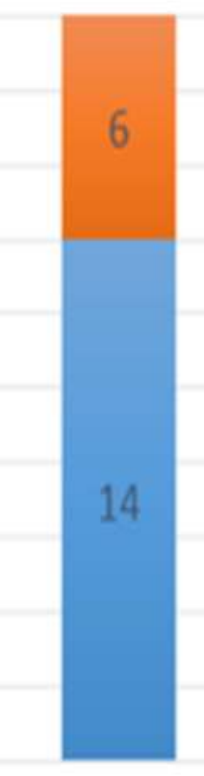

$10 \%$ hypertonic $30 \%$ hypertonic saline saline

- Survival = Death

\section{Background:}

Brain swelling is the most feared complication of malignant stroke with massive brain infarction. The high mortality of such complications is directly related to intracranial herniation due to swelling. Decompressive craniectomy is usually regarded as a last resort of treatment. Hypertonic saline has been favorably used as a hyperosmolar agent for the past decades in various intracranial pathological conditions. In this study, we evaluated hypertonic saline in the treatment of such conditions in rat model of malignant stroke. Our primary end point was 24-hr mortality, and our secondary end point was brain water content

Material and methods:

A total of 45 adult male rats were studied with a catheter implanted in the internal carotid artery. After discontinuation of isoflurane anesthesia, a clot was injected into the internal carotid artery with the rats conscious and freely moving in their home cage. The rats were divided into three groups to receive normal saline (Group 1 control) or $36 \mathrm{mOsm} / \mathrm{kg}$ solution of either $10 \%$ hypertonic saline (50-50 chloride-acetate; Group 2) or 30\% hypertonic saline (Group 3). Treatment consisted of a 60 -min loading beginning 5 min after embolization and followed 300 mins later by a $34 \mathrm{mOsm} / \mathrm{hr}$ infusion of the same solution for $22 \mathrm{hrs}$. Twenty-four hours after embolization, blood was sampled for measurement of sodium and osmolality. Rats were then killed under deep anesthesia, and the brain was harvested for measurement of water content. Brain water contents were derived by wet/dry weight ratio.

\section{Results:}

Twenty-four-hour mortality was significantly reduced in both hypertonic saline treated groups (NS = 58\%; $10 \% \mathrm{HS}=30 \%$; $30 \% \mathrm{HS}=25 \%, \mathrm{p}<0.05)$. Twenty-four hours after embolization, the water content on the infarcted hemisphere was unchanged for those that survived in NS or HS treated rats (NS $=83.1 \pm 1.1 \%$; $10 \% \mathrm{HS}=83.0 \pm 1.1 \% ; 30 \% \mathrm{HS}=83.8 \pm 0.7 \%$ ), whereas the HS-treated contralateral hemisphere was significantly decreased (NS = $79.1 \pm 0.6 \% ; 10 \% \mathrm{HS}=77.4 \pm 0.8 \% ; 30 \% \mathrm{HS}=77.4 \pm 0.6$ $\% ; p<0.05)$. Serum sodium was unchanged in NS-treated rats, but increased significantly in HS-treated rats $(10 \% \mathrm{HS}=151.8 \pm 6.5$ $\mathrm{mEq} / \mathrm{L} ; 30 \% \mathrm{HS}=155.5 \pm 6.6 \mathrm{mEq} / \mathrm{L}, \quad \mathrm{p}<0.05)$. Likewise, serum osmolality was unchanged in NS treated rats, but increased significantly in $\mathrm{HS}$ treated rats $(10 \% \mathrm{HS}=347.5 \pm 11.6 \mathrm{mOsm} / \mathrm{L}$; $30 \%$ HS $353.6 \pm 10.3 \mathrm{mOsm} / \mathrm{L}, \mathrm{p}<0.05)$.
24 Hours, Right and Left Hemisphere Brain Water Content in Survived and Dead Rats Following a Single Blood Clot Injection to ICA

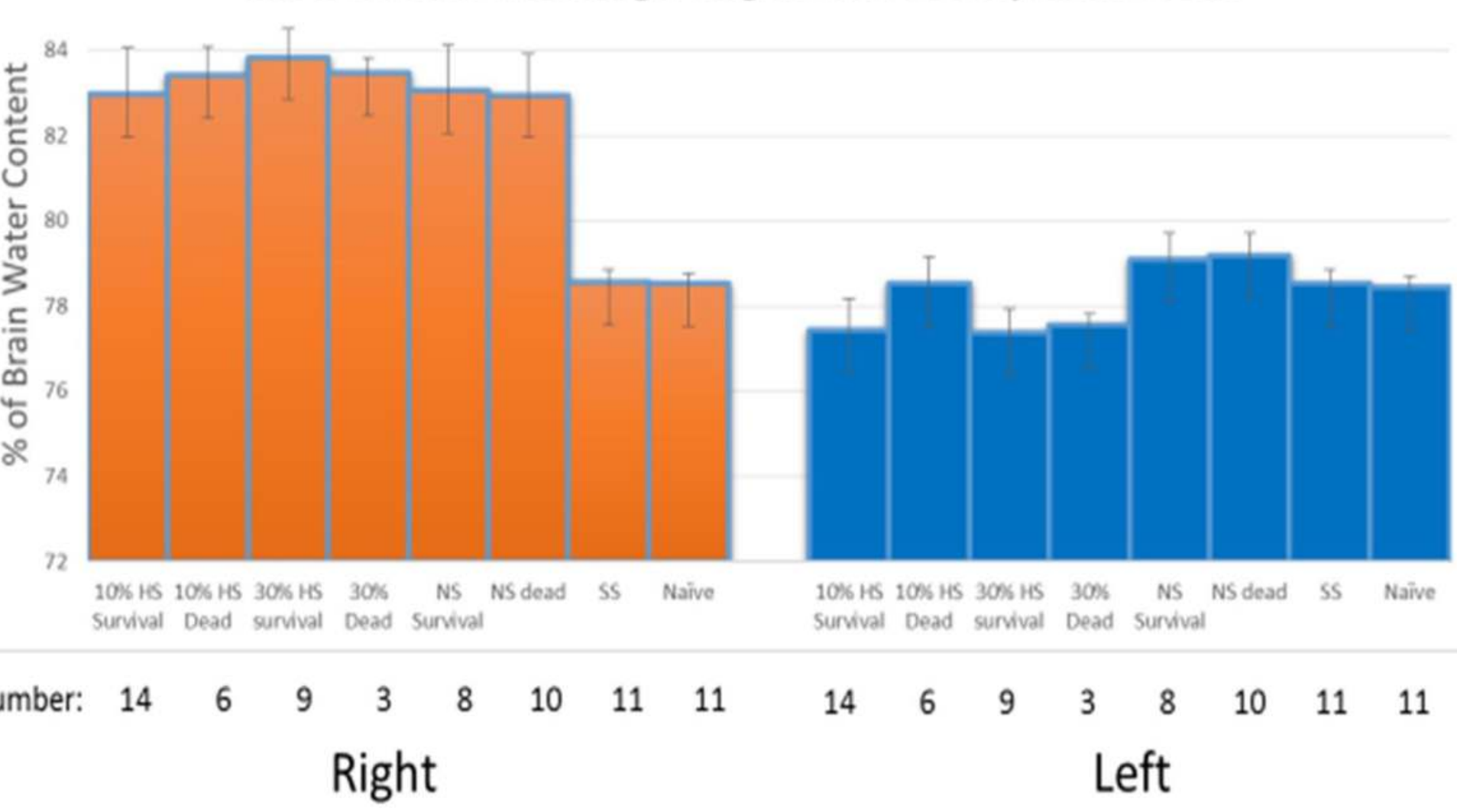

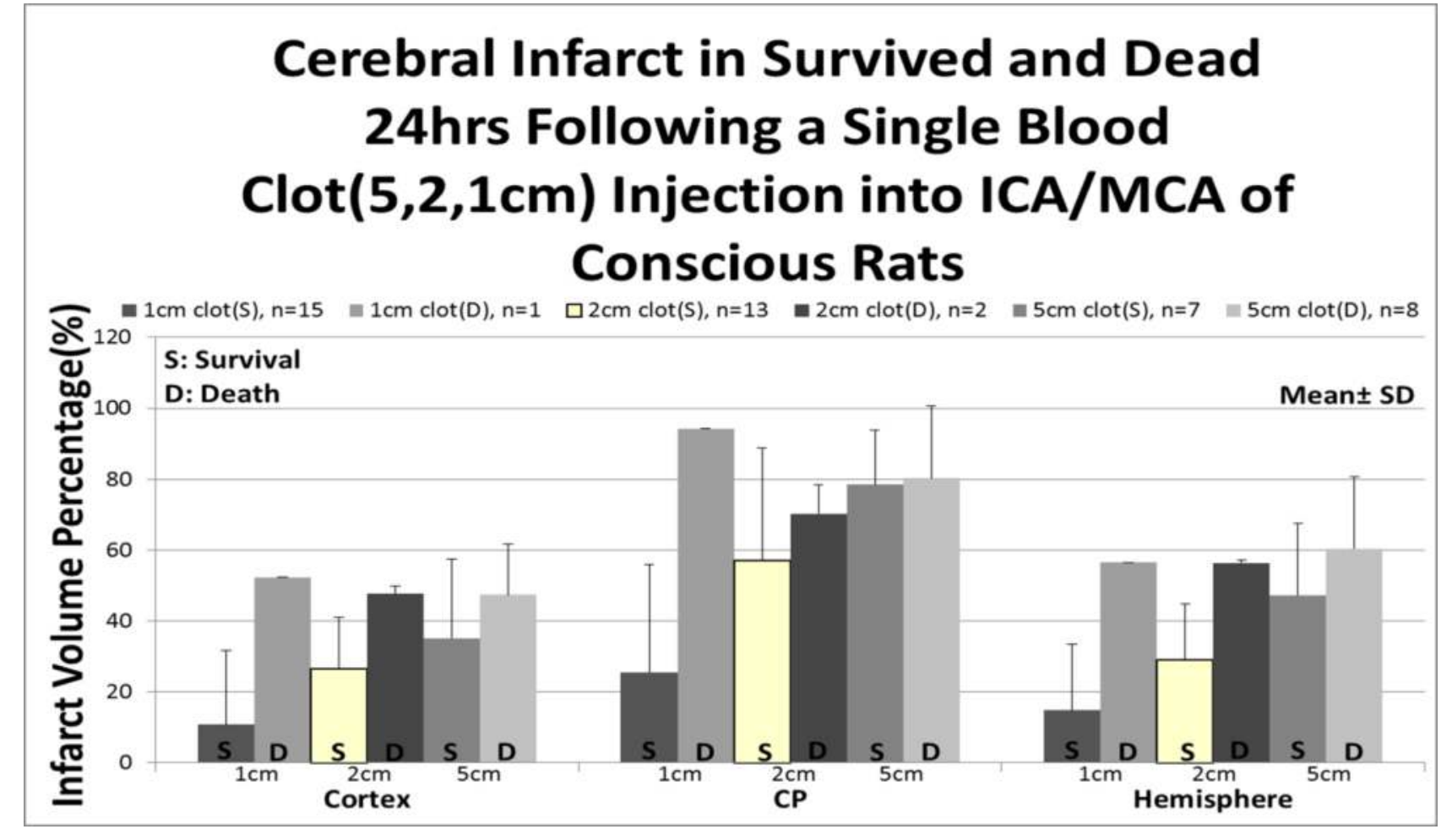

Cerebra Edema Time Course After Clot Injection in Stroke Rat Model

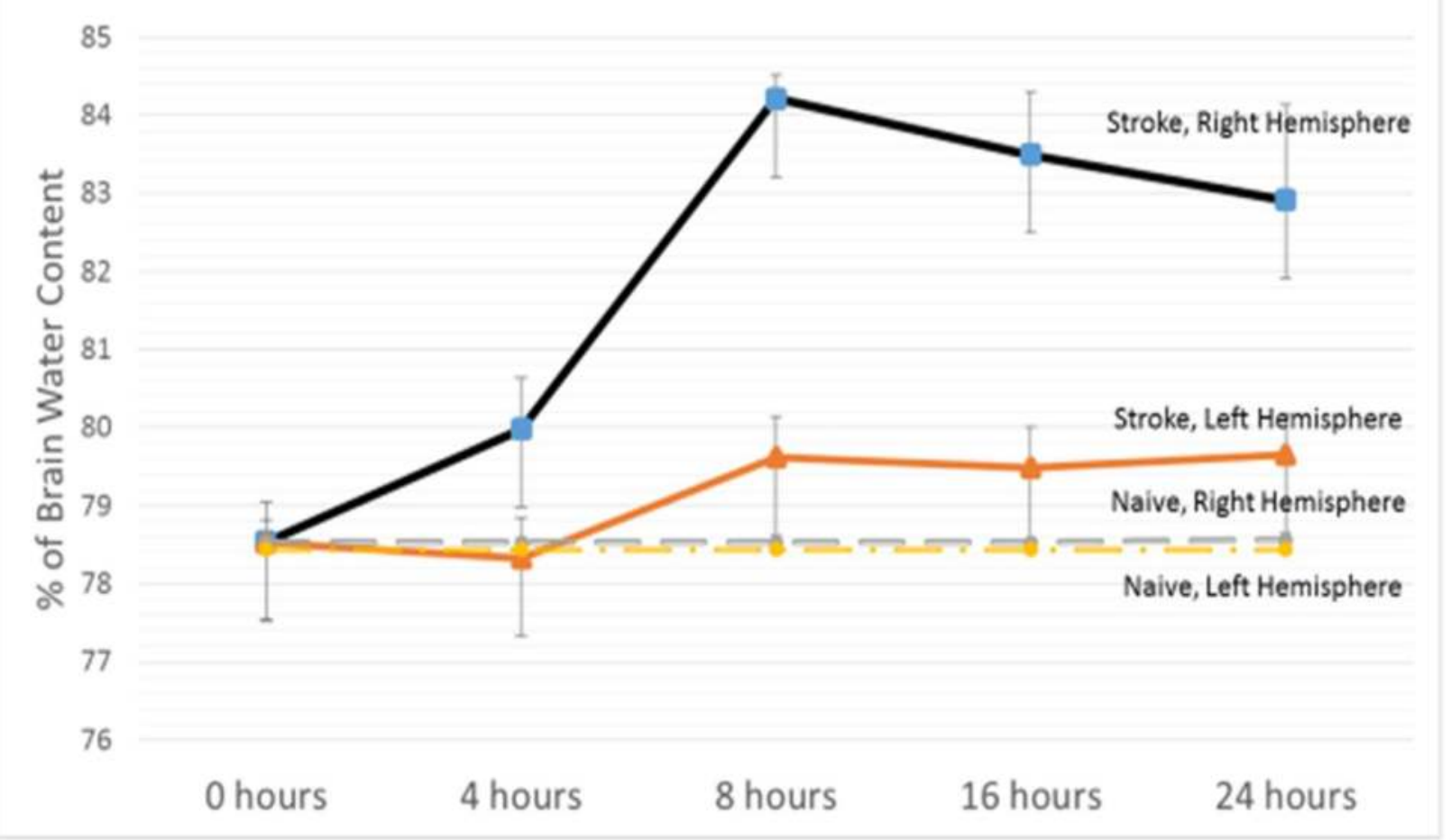

Conclusion:

HS reduced contralateral hemispheric water content but did not affect ipsilateral brain water content when compared to NS. This difference was most likely due to a higher sodium permeability across a leaky blood-brain barrier in the ischemic hemisphere. Nevertheless, the reduced contralateral brain water content was associated with decreased mortality. We conclude that sustaining a hyperosmolar state for one day with hypertonic saline is effective in reducing mortality following experimental malignant embolic stroke. 